\title{
Classical Fourier Analysis Applied to a Simple Out- line: The Bovine Obturator Foramen
}

\author{
P.-M. Parés i Casanova, I. Kucherova \\ Dept. of Animal Production, University of Lleida. Av. Alcalde Rovira Roure, 191. \\ E-25198 Lleida (Catalunya) SPAIN \\ peremiquelp@prodan.udl.cat
}

With I table and 2 figures

Received December 2011, accepted for publication January 2012

\section{Abstract}

The purpose of this article it to present a procedure of shape characterisation using the classical Fourier analysis combined with image techniques. It was applied to the outline of the obturator foramen of the bovine pelvis. This outline was extracted and characterised by an ordered series of harmonics, each harmonic being described by four coefficients. Step by step reconstructions of outlines using an increasing number of harmonics were then performed. Although the first seven harmonics reached $93.4 \%$ of the cumulative power, the shape of the obturator foramen was already well described by the first harmonic. It seems that the classical Fourier analysis opens interesting perspectives for the study of simple osseous outlines.

\section{Kew words}

J. Vet. Anat. image analysis; morphometry; pelvis; bovine anatomy

\section{Introduction}

The shape evaluation is a powerful tool for analysing biological shapes. Fourier method have been developed and applied in morphometry in order to characterise outlines (Bierry et al., 2010; Schmittbuhl et al., 2002). Simple outlines are characterised by the fact that the radii emanating from the centre of the area intersect the outline once and only once. Fourier analysis has several advantages over other methods. This analysis is a mathematical way of reducing complex curves (McLellan and Endler, 1998) and allows a complete quantification of outlines without the necessity for determination of precise landmarks. Moreover, it provides a precise and accurate description of shape over a range of size scales (Kuhl and Giardina, 1982; Kincaid and Schneider, 1983). Therefore it 
seems appropriate for the analysis of outlines such as large bony foramina in which there is a clear difficulty to localise precise and repeatable reference points. Moreover, the Fourier methods are considerably facilitated due to major advances in computer science.

The obturator foramen is a large opening in the hip bone, which is bridged by the obturator membrane, except for above, where there is a communication between the pelvis and the thigh. This communication, also known as the obturator canal, is the passage through which the obturator vessels and nerves pass (Standring, 2005). Shape analysis has been scarcely applied to the study of the obturator foramen (Bierry et al., 2010; Ridgeway et al., 2008), although it is of great interest, for instance for sex determination from archaeological remains (Ruscillo, 2003). There is also a paucity of literature on the anomalies of the obturator foramen (Das et al., 2006).

The purpose of this article is to present an easy procedure of shape characterisation using classical Fourier methods combined with image analysis techniques in order to evaluate the suitability of this analysis.

\section{Material and methods}

J. Vet. Anat.

\section{Specimen preparation}

Fifty five bovine Ossa coxae free of pathologic changes were used. Both left and right Ossa coxae were sampled. All specimens were obtained from the commercial abattoir MAFRISEU SA in la Seu d'Urgell (Catalunya, Spain).

\section{Image capture and processing}

Image capture was performed with a Nikon $®$ D70 digital camera (image resolution of $2240 \times 1488$ pixels) equipped with a Nikon AF Nikkor 28-20 mm® telephoto lens. The focal axis of the camera was parallel to the horizontal plane of reference, and centred on the obturator foramen. For scaling purposes a ruler was concurrently photographed on each image. The visualisation and processing of the images were made with the software package GIMP v. 2.6.11®. After the outline of interest was manually extracted, a threshold transformation of the greylevel image into a binary image was applied.

\section{Fourier Analysis}

Fourier analysis was performed with Shape software (Iwata and Ukai, 2002). This program provides image analysis, contour recording (shape), derivation of the Fourier descriptors (Chc2Nef) and principal component analysis on the descriptors. An outline could be described by an or- 
dered series of harmonics (Fourier shape components). Four coefficients were produced for each harmonic. The outline was composed of 1,852 points (the number of harmonics requested must be constrained to be equal to or less than the number of points divided by 2). As in general Fourier expansions of shape data are sensitive to information that is usually considered irrelevant, such as location, size and orientation of the object, a normalisation has been applied.

\section{Fourier reconstructions}

Step by step reconstructions of outlines were performed using an increasing number of harmonics. The cumulative power was calculated for each step of the reconstruction. Reconstructions were made point-bypoint and corresponded to a summation of vectors, each vector having its origin on the centre of the corresponding harmonic and its extremity on the contour considered.

\section{Statistical analysis}

The Fourier coefficients were calculated. The evaluation of accumulative power was performed using a principal component analysis.

\section{Results}

The summary statistics of the Fourier coefficients of the outline of the obturator foramen are listed in Table I. Only the first 7 harmonics were retained in this table. Step by step reconstructions of the outline are represented in Figure 1. The accuracy of the reconstructions increased with the number of harmonics used. Reconstruction based on the first harmonic resembles the original outline closely. The accumulative power between the reconstructed and the original outlines reached $81.3 \%$ by using the first three harmonics and was superior to $93 \%$ by using the first seven (Figure 2).

\section{Discussion}

Step by step reconstructions allow interpretation of the geometric contribution of each Fourier harmonic. As expected, the coefficients of the lower order Fourier harmonics correspond to the overall shape, and the higher order harmonics correspond to smaller details of the outline (Kuhl and Giardina, 1982; Kincaid and Schneider, 1983). In the present application, the first seven harmonics were sufficient for a precise description of the entire obturator foramen outline; they describe the total shape and form of the foramen. Ulterior harmonics do not add finer morphological features. Computer-assisted image analysis used in the present study allowed a 
quantification of the shape of outlines of interest. It presented numerous advantages compared with classical biometric analysis, i.e. greater objectivity and reproducibility, greater rapidity, and facilitation of measurements traditionally impossible to determine directly. Another advantage of image analysis is the possibility of creating image databases for archiving the original images, and metric databases facilitating statistical interpretation. Fourier descriptors provide a quantification of the shape of an outline, whatever its morphological complexity. New developments in Fourier methods provide a new set of parameters, whose relation to a geometric interpretation is considerably easier than classically. The set of descriptors determined by the present method provides an individual characterisation or geometric signature of the outline studied. Fourier descriptors also constitute a very powerful tool for data compression. Using the present easy methodology, a precise comparison of a series of outlines becomes easier and more reproducible. Further studies, based on sex comparisons, will elucidate if there are sex differences in morphology of obturator foramen. These new developments in Fourier methods and the present methodology can of course be applied to all bidimensional outlines.
Although other morphometric methods such as traditional morphometric multivariate analysis of linear measurements and geometric morphometrics can be used, classical Fourier analysis permits the automation of the image analysis and consequently a greater reproducibility, and above all, an easy use even by a non-specialist. Moreover, landmarks points can be difficult to standardise in a hole as the obturator foramen is. The developed software is moreover adapted for a personal microcomputer, and is thus easily communicable and transportable. Elliptic Fourier analysis is a more elaborate method allowing the quantification of complex outlines, but it is mathematically complex and this could explain why it has been rarely applied.

\section{References}

Bierry G, Le Minor J-M, Schmittbuhl M. (2010): Oval in males and triangular in females? A quantitative evaluation of sexual dimorphism in the human obturator foramen. American Journal of Physical Anthropology 141:626-631.

Das S, Suri R, Kapur V. (2006): A triplicate obturator foramen. Folia Morphologica 65(2):164166.

Iwata H, Ukai Y. (2002): SHAPE: A 
computer program package for quantitative evaluation of biological shapes based on elliptic Fourier descriptors. Journal of Heredity 93: 384-385.

Kincaid DT, Schneuider RB. (1983):

Quantification of leaf shape with a microcomputer and Fourier transformation. Canadian Journal of Botany 61:23332342.

Kuhl FP, Giardina CR. (1982): Elliptic Fourier features of a closed contour. Computer Graphics and Image Processing 18:236258.

McLellan T, Endler JA. (1998): The relative success of some methods for measuring and describing the shape of complex objects. Systematic Biology 47(2):264-281.

Ridgeway BM, Arias BE, Barber

MD. (2008): Variation of the obturator foramen and pubic arch of the female bony pelvis. American Journal of Obstetrics \& Gynecology 198(5):546.e1546.e4.

Ruscillo D. (2003): Alternative methods for identifying sex from archaeological animal bone. In: Kojabopoulou E, Hamilakis $\mathrm{Y}$, Halstead $\mathrm{P}$, Gamble C, Elefanti P (eds.). Zooarchaeology in Greece. Recent Advances. BSA Stud- ies 9. The British School at Athens. London: 37-44.

Schmittbuhl M, Le Minor J-M, Schaa A, Mangin P. (2002): The human mandible in lateral view: elliptical fourier descripttors of the outline and their morphological analysis. Annals of Anatomy - Anatomischer Anzeiger 184(2):199-207.

Standring S. (2005): Gray's anatomy. The anatomical basis for clinical practice. 39th Ed., Elsevier Churchill Livingstone, London, pp. 1422.

\section{Acknowledgements}

Sincere thanks are expressed to MAFRISEU SA, which provided the samples. 
Fourier Analysis of Bovine Obturator foramen

Table (I): Eigenvalues and cumulative proportions of the outline of the obturator foramen of the bovine pelvis. Results for the first 7 principal components (PC).

\begin{tabular}{|c|cccc|}
\hline & Eigenvalue & Proportion (\%) & Cumulative (\%) & $>1 / 77$ \\
\hline PC1 & $2.62 \mathrm{E}-03$ & 40.572 & 40.572 & $*$ \\
PC2 & $2.01 \mathrm{E}-03$ & 31.143 & 71.716 & $*$ \\
PC3 & $6.20 \mathrm{E}-04$ & 9.585 & 81.301 & $*$ \\
PC4 & $3.24 \mathrm{E}-04$ & 5.014 & 86.315 & $*$ \\
PC5 & $2.49 \mathrm{E}-04$ & 3.845 & 90.160 & $*$ \\
PC6 & $1.11 \mathrm{E}-04$ & 1.710 & 91.871 & $*$ \\
PC7 & $9.97 \mathrm{E}-05$ & 1.542 & 93.413 & $*$ \\
\hline
\end{tabular}

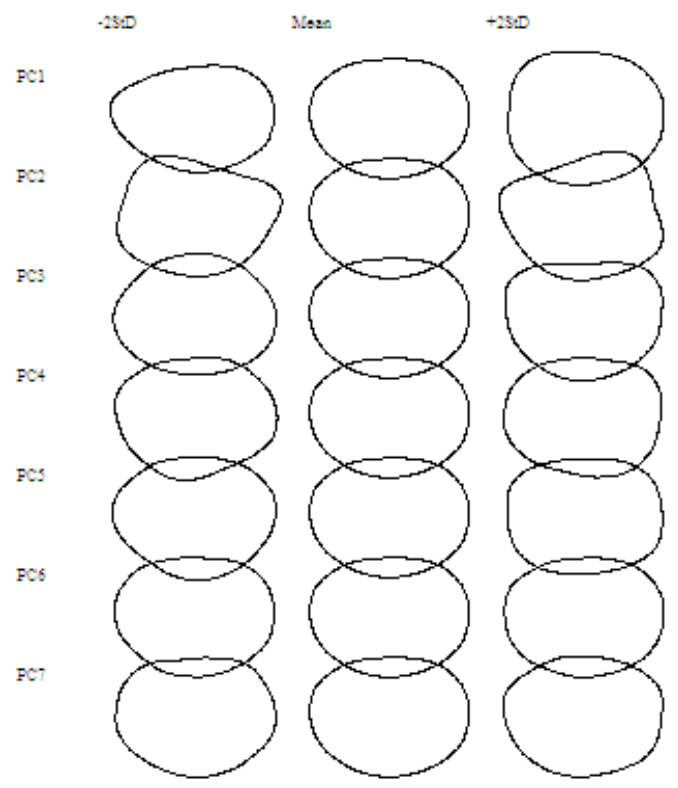

Fig (1): Step by step reconstructions of the distal obturator foramen outline (mean \pm Standard Deviation) by addition of the first seven harmonics. 


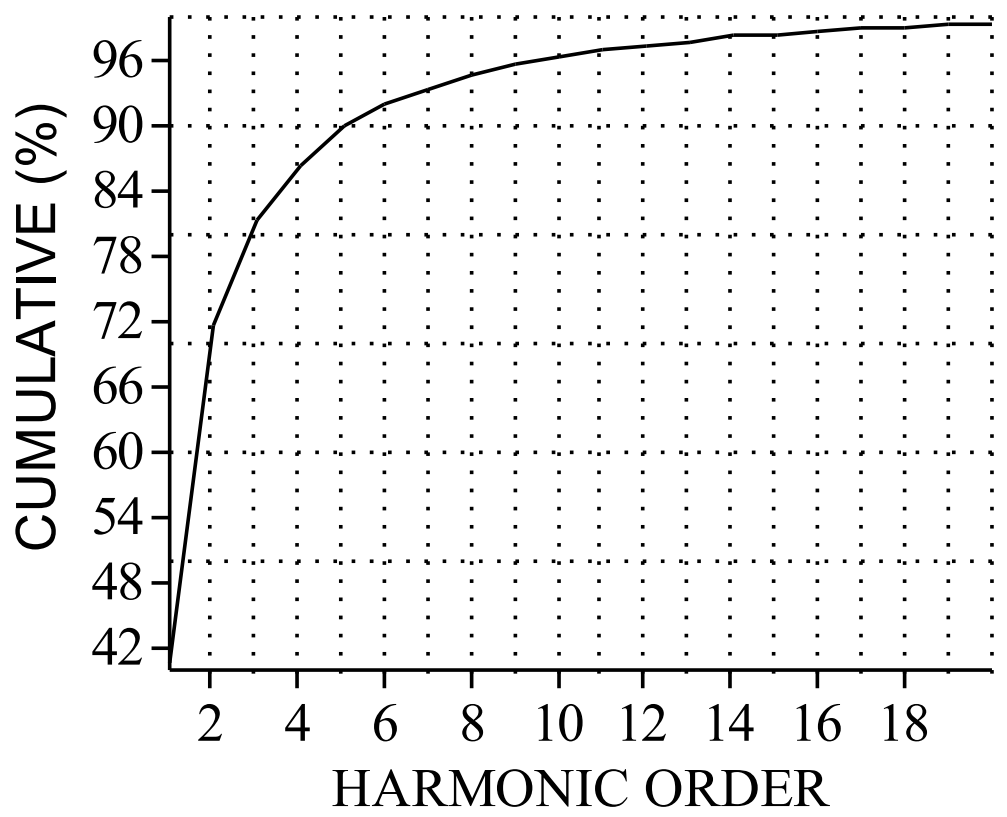

Fig (2): Step by step reconstructions of the outline of the obturator foramen of the bovine pelvis for the first 20 harmonics. 


\section{Animal species in this issue}

\section{Domestic goat (Capra aegagrus hircus)}

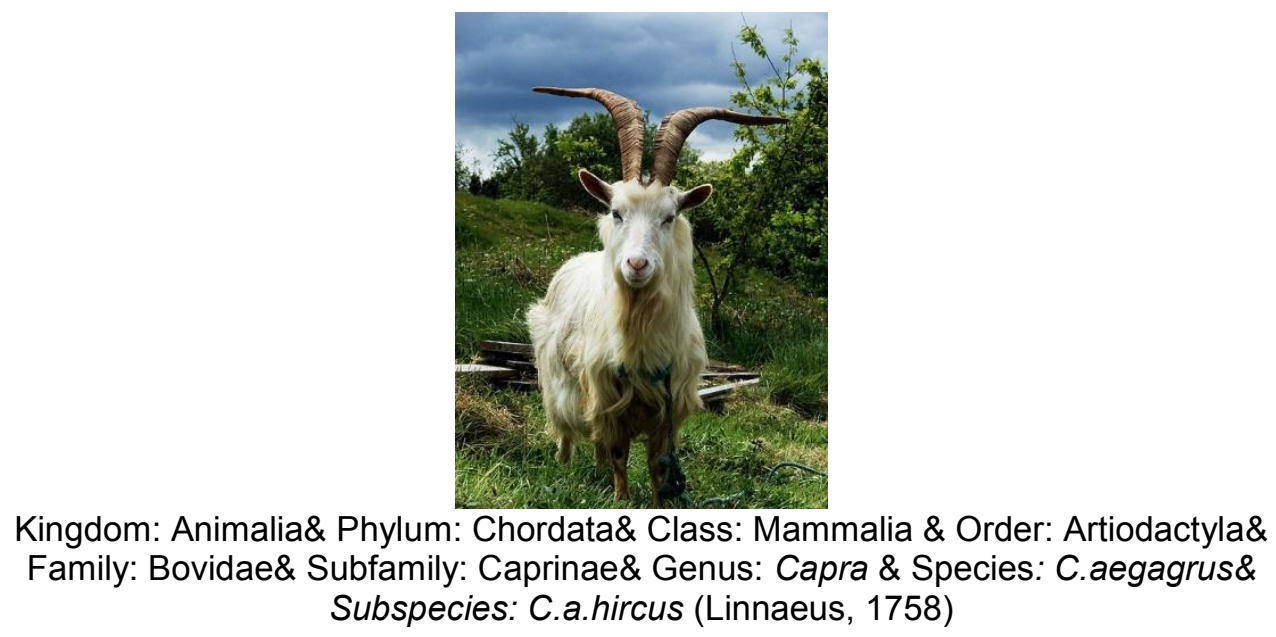

The domestic goat (Capra aegagrus hircus) is a subspecies of goat domesticated from the wild goat of southwest Asia and Eastern Europe. The goat is a member of the Bovidae family and is closely related to the sheep as both are in the goat-antelope subfamily Caprinae. There are over three hundred distinct breeds of goat.

Female goats are referred to as does or nannies, intact males as bucks or billies; their offspring are kids. Note that many goat breeders prefer the terms "buck" and "doe" to "billy" and "nanny". Castrated males are wethers. Goat meat from younger animals is called kid or cabrito, and from older animals is sometimes called chevon, or in some areas "mutton".

Goats are ruminants. They have a four-chambered stomach. The females have an udder consisting of two teats, in contrast to cattle, which have four teats.

Goats have horizontal slit-shaped pupils, an adaptation which increases peripheral depth perception. Because goats' irises are usually pale, the pupils are much more visible than in animals with horizontal pupils, but very dark irises, such as cattle, deer, most horses and many sheep. Both male and female goats have beards, and many types of goat (most commonly dairy goats, dairycross boers, and pygmy goats) may have wattles, one dangling from each side of the neck. 